\title{
VY Sculptoris stars are Magnetic Cataclysmic Variables
}

\author{
Jean-Pierre Lasota \\ UMR 7095 du CNRS, Institut d'Astrophysique de Paris, \\ 98bis Boulevard Arago, 75014 Paris, France \\ Jean-Marie Hameury \\ UMR 7550 du CNRS, Observatoire de Strasbourg, \\ 11 rue de l'Université, F-67000 Strasbourg, France
}

\begin{abstract}
We show that VY Scl stars must be magnetized in order to account for the absence of outbursts during their low and intermediate states. Absence of outbursts during low states requires only rather low magnetic moments but in systems in which the drops and rises of luminosity are slower than it takes for the accretion disc to adjust viscously to the variation in mass-transfer rate preventing outbursts require magnetic moments of Intermediate Polars. We discuss some evolutionary aspects of this conclusion.
\end{abstract}

\section{Introduction}

VY Scl stars are very bright Nova Like (NL) Cataclysmic Variables (CVs) which occasionally undergo a fall in brightness by more than one magnitude. Such drops in luminosity, which seem to result from a decrease of the mass-transfer rate, should bring the accretion discs in these systems into the Dwarf Nova (DN) instability strip, but surprisingly no outbursts are observed. In general, light-curves of VY Scl stars show no activity at all during descents and rises and although low states are sometimes interrupted by epochs of activity their characteristic time-scales are much longer than those of the thermal-viscous instability. Clearly the instability triggered by hydrogen recombination which is at the origin of dwarf-nova outbursts is not operating in these systems.

The question of outburst absence in VY Scl stars can be divided into two parts: 1. Why is there no outbursts during low states?, and 2. Why is there no outbursts during long intermediate states (descents and rises)? By "long" we mean longer than the disc's viscous time. Indeed, the disc behaviour will depend on how fast the mass-transfer rate is switched off (on).

\section{Absence of outbursts during low-states}

Let us first consider the case when the mass transfer rate is stopped instantaneously (which means in a time shorter than the viscous time). Since no matter will be arriving to the disc's outer edge anymore, its surface density will drop un- 
til it reaches the critical density $\Sigma_{\min }$ at which no hot steady solutions exist (see e.g. Lasota 2001). As in, at dwarf-nova maximum, when the quasi-stationary disc accretes at a rate much higher than the rate at which matter is transferred from the secondary, a cooling (rarefaction) wave will propagate inwards leaving behind a non-steady disc in which the surface density (and the accretion rate) increases with radius. Therefore, even with no transfer of mass, matter will diffuse inwards and the surface density will increase near the inner disc edge. Since the critical surface density $\Sigma_{\max }$ strongly decreases with radius the disc will inevitably enter the parameter range in which no thermal-equilibrium solution exist, i.e. it will go into outburst.

As noticed by Lasota, Hameury \& Huré (1995) one would prevent an outburst if the disc were truncated at radius

$$
r_{\text {tr }}>r_{\text {crit }} \approx 6 \times 10^{9}\left(\frac{\dot{M}}{10^{15} \mathrm{~g} \mathrm{~s}^{-1}}\right)^{0.375} M_{1}^{0.333} \mathrm{~cm},
$$

where $r_{\mathrm{tr}}$ is the transition radius and $M_{1}$ is the white-dwarf mass in solar unit (see e.g. Lasota 2001); e.g. a (cold) disc truncated at $r_{\mathrm{tr}} \gtrsim 6 \times 10^{9} \mathrm{~cm}$ would be stable for accretion rates lower than $10^{15} \mathrm{~g} \mathrm{~s}^{-1}$.

Leach et al. (1998) tried to explain the absence of outbursts during VY Scl low-states by assuming that such truncation can be achieved by irradiating the inner disc by the white-dwarf which in VY Scl stars is rather hot (e.g. Sion 1999). The quiescent disc would thus have two components: very hot $\left(T_{\text {eff }}>7000 \mathrm{~K}\right)$ for $r<r_{\mathrm{tr}}$; very cold $\left(T_{\mathrm{eff}}<3000 \mathrm{~K}\right)$ for $r>r_{\mathrm{tr}}$. Unfortunately, Leach et al. (1998) tested their idea only in a system with a very low mass $\left(0.4 \mathrm{M}_{\odot}\right)$ white dwarf. By a strange coincidence this is the only case where their idea could work.

Indeed, the white-dwarf irradiation temperature is given by (e.g. Hameury, Lasota \& Dubus 1999)

$$
T_{\mathrm{irr}}^{4}=(1-\beta) T_{*}^{4} \frac{1}{\pi}\left[\arcsin \rho-\rho\left(1-\rho^{2}\right)^{1 / 2}\right]
$$

where $\rho=R_{*} / r, R_{*}$ and $T_{*}$ are the white dwarf radius and temperature. $1-\beta$ is the fraction of the incident flux which is absorbed in optically thick regions, thermalized and re-emitted as photospheric radiation. The transition radius between the hot and cold disc is approximately

$$
r_{\mathrm{tr}} \approx 6.7 T_{*, 40}^{4 / 3}\left(\frac{T_{\text {crit }}}{6500 K}\right)^{-4 / 3} R_{*}
$$

where we put $\beta=0$ (no albedo, which obviously maximizes the transition radius) and assumed for simplicity that a disc is thermally stable if its photospheric temperature is larger than $T_{\text {crit }} \simeq 6500 \mathrm{~K}$. $\left(T_{*, 40}=T_{*} / 40,000 \mathrm{~K}\right)$. A comparison with Eq. (1) shows immediately the advantage of using a $0.4 \mathrm{M}_{\odot}$ white dwarf, for which $R_{*}=1.07 \times 10^{9} \mathrm{~cm}$. A $0.7 \mathrm{M}_{\odot}$ would stabilize the disc only if heated to more than $50,000 \mathrm{~K}$. A convincing observational confirmation of this conclusion is provided by the cataclysmic variable RU Peg. It has the hottest white dwarf 


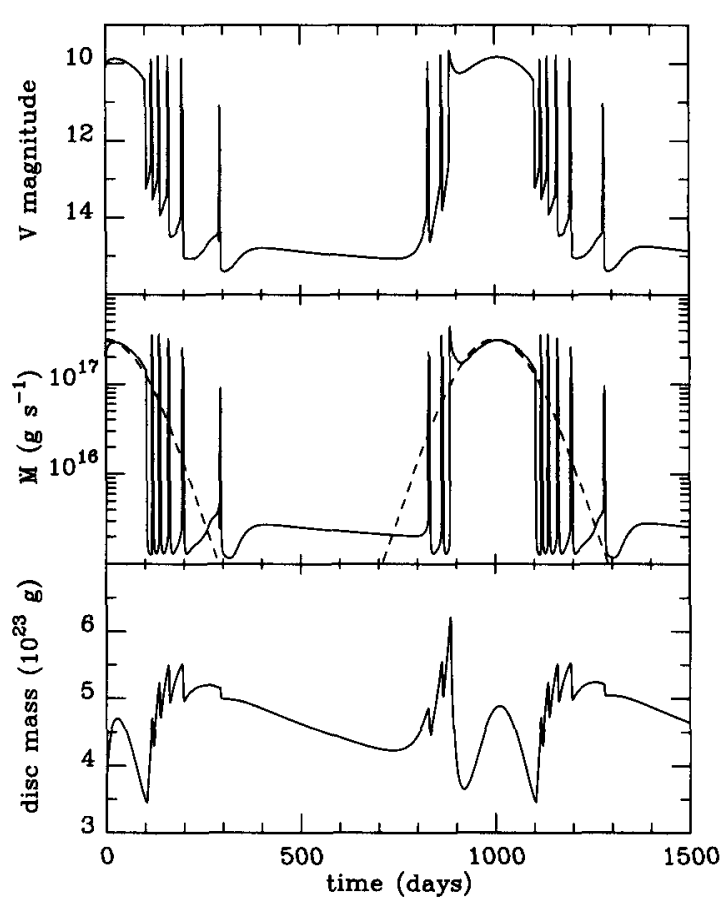

Figure 1. Top panel: visual light curve of a binary system in which the mass-transfer rate slowly varies. The accretion disc is disrupted by the magnetic field of a $0.7 \mathrm{M}_{\odot}$ white dwarf with $\mu_{30}=10$. Intermediate panel: mass accretion rate onto the white dwarf (solid line), and mass transfer rate (dashed line). Bottom panel: disc mass

observed (50 $000 \mathrm{~K}$; Sion, \& Urban $\left.2002^{1}\right)$... but it is a dwarf nova. The white dwarf mass is $\sim 1.2 \mathrm{M}_{\odot}$.

As showed by Hameury \& Lasota (2003) sufficient truncation can be achieved if the white dwarf has a rather weak magnetic moment of $\mu=5 \times 10^{30} \mathrm{Gcm}^{3}$. VY Scl stars with such white-dwarf's magnetic fields would show no outbursts during low states.

\section{Absence of outbursts during intermediate states}

However, such truncation would not prevent outbursts during long intermediate states. Indeed, truncation may suppress "inside-out" outbursts, i.e. out-

\footnotetext{
${ }^{1}$ These authors try to fit the spectrum of a quiescent dwarf nova with a stationary disc model. Not surprisingly (quiescent discs are non-steady) this does not work, but their white-dwarf model (giving $50,000 \mathrm{~K}$ ) is consistent with the dwarf-nova model
} 
bursts starting near the inner edge of the disc (see Lasota 2001). The fate of "outside-in" outbursts (starting in the outer disc regions) depends on how the mass-transfer is varied: an outburst is of outside-in type if the accumulation time of transferred matter $\Delta M / \dot{M}_{\text {transf }}$, where $\Delta M$ is the mass accumulating in the outer disc and $\dot{M}_{\text {transf }}$ is the mass-transfer rate, is shorter then the viscous (diffusion) time. When, for example, the mass transfer is switched off instantaneously, the accumulation time becomes infinite and no outside-in outburst is possible. If on the other hand the mass transfer rate varies very slowly, with a characteristic time-scale much longer than the disc's viscous time, the disc will enter the instability regime in a quasi-stationary way and outside-in outbursts are unavoidable (Fig. 1). For time-scales between the two, either of the two types is possible depending on the way the mass-transfer is changing. The only way to avoid outbursts is to get rid of the disc itself. No disc, no outbursts.

As a "no-disc" condition we can require, for example, that when the mass transfer rate from the secondary drops below the critical value corresponding to the end of hot branch at the outer disc's edge, $\dot{M}_{\text {crit }}\left(r_{\text {out }}\right)\left(r_{\text {out }}\right.$ being the outer disc radius), the magnetospheric radius becomes larger than the circularization radius. As showed by Hameury \& Lasota (2003) one obtains then the following general "no-disc" condition in terms of the required magnetic moment:

$$
\mu \gtrsim 1.5 \times 10^{33} f_{0.12}^{1.75} P_{4}^{2.06}\left(3 r_{\text {out }} / a\right)^{1.34} M_{1}^{1.4} \mathrm{G} \mathrm{cm}^{3}
$$

where a typical mass ratio $M_{2} / M_{1}=0.43$ is assumed, the value $f=0.12$ corresponds to the circularization radius in units of the binary separation $a$, the orbital periods measured in units of 4 hours, and the outer radius $r_{\text {out }}$ normalized by its typical value $a / 3$.

Numerical simulations show that this is an overestimate, the critical magnetic moment being about half the value deduced from Eq. (4). This is due to the fact that heating by the tidal torque and stream impact has a stabilizing effect in the outer disc regions, an effect that has not been taken in account when deriving Eq. (4). Also $r_{\text {out }}$ decreases significantly when the inner disc radius approaches $r_{\text {circ }}$. Therefore the no-disc condition for VY Scl requires magnetic moments in the range

$$
\mu \gtrsim 4-7.5 \times 10^{32} \mathrm{G} \mathrm{cm}^{3}
$$

i.e. VY Scl should have magnetic moments of Intermediate Polars (IP).

Our model has been successfully tested in the case of RX And. This cataclysmic variable is the answer ("here") to Hellier \& Naylor's (1998) question: "... why is there no transitional objects between ZC and VY?". RX And is half Z Cam, half a VY Scl star (Schreiber et al. 2002). Since it is a dwarf nova during long stretches of its life, the magnetic moment of its white dwarf cannot satisfy Eq. (4) as a disc is needed to produce outbursts. This is confirmed by the absence of long intermediate state with no outbursts: VY Scl-type brightness drops and rises are fast and when the system settles to a long intermediate state below the standstill luminosity shows outburst activity. The standstill itself corresponds to a stable mass-transfer rate (Buat-Ménard et al. 2001). A system exhibiting both dwarf-nova outbursts and long and quiet luminosity descents below the stability limit would contradict our model and presumably the disc instability model in general. 


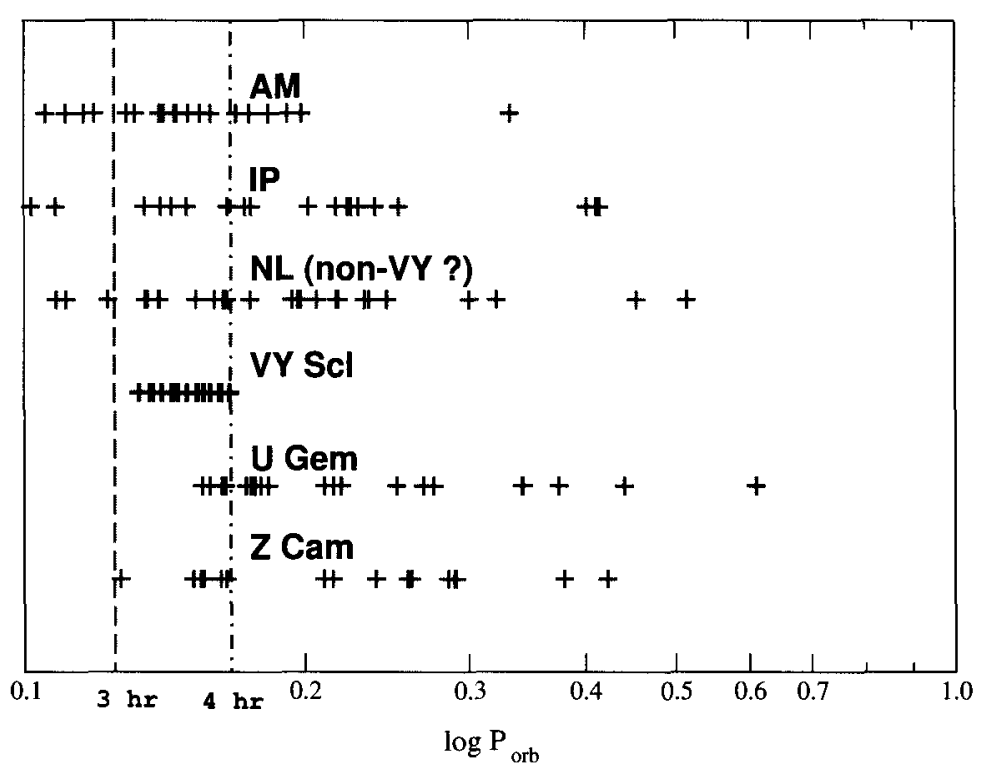

Figure 2. Orbital period distribution of various classes of CVs between 2.4 and 15 hours. See Hameury \& Lasota (2003) for the references.

\section{Relation to other systems}

The conclusion that at least some of the VY Scl stars are magnetic cataclysmic variables seems therefore unavoidable. This is not an extravagant conclusion, on the contrary: it seems to fit to a rather fashionable trend inspired by arguments independent of ours. For example Patterson et al. (2003) argue that SW Sex stars ("SW Sexers") are "borderline AM Herculis stars, usually drowned by a high accretion rate". This conclusion is based on the analysis of periodic and quasi-periodic signals, especially superhumps, of two SW Sex binaries: V442 Oph $P_{\text {orb }}=2.98392 \mathrm{hr}$ and RX J1643+3492 $P_{\text {orb }}=2.89344$. "SW Sexers" are nova-like CVs showing anomalous phenomena in their emission lines, where "anomalous" means different from the simple binary model of CVs (e.g. singlepeaked emission lines, large phase lags etc.). Also their continua show unusual temperature profiles. These anomalies are detected in systems with high inclinations, but seem, in fact, to be shared by most CVs at high accretion rates (Horne 1999). Four VY Scl stars (LX Ser, BH Lyn, DW UMa and PG And) are, when in maximum, SW Sex stars. Patterson et al. (2003) find interesting superhump behaviour (changing signs) in DW UMa.

In two (non VY Scl) SW Sex stars, LS Peg (Rodríguez-Gil et al. 2001) and V795 Her (Rodríguez-Gil et al. 2002), variable circular polarization $(\sim 0.3 \%)$ has been discovered. The authors of these observations deduce magnetic fields 
of 5-15 MG (LS Peg) and 2-7 MG (V795 Her). The circular polarization in LS Peg and V795 Her is modulated with periods of $\sim 20-30$ min, typical of IPs and fitting the $P_{\text {spin }} / P_{\text {orb }} \sim 0.1$ relation (see e.g. King \& Lasota 1991).

Tovmassian (private communication) found that some unusual properties of the VY Scl star V751 Cyg could be convincingly explained by the presence of a magnetized white dwarf.

Patterson et al. (2003) speculate that SW Sex systems could be ancestors of AM Her stars, spun up to kilosecond periods. Their magnetic properties would be "drowned" by high accretion rates. If it were the case of e.g. the VY Scl star DW UMa its magnetic properties such as circular polarization should be observable during the low states (it is not clear, however, that such relevant observations have been performed). Other difficulties associated with the AM Her hypothesis for SW Sex stars are extensively discussed in Patterson et al. (2003).

Our "no-disc" condition, although compatible with AM Her-type magnetic moments (the condition is an inequality), requires weaker fields and VY Scl would rather be highly accreting IPs. In fact the mean mass-transfer rate for IPs is $\dot{M}_{\text {transf }}^{I P} \lesssim 2 \times 10^{17} \mathrm{~g} \mathrm{~s}^{-1}$, while for VY Scl stars $\dot{M}_{\text {transf }}^{V Y} \sim 2-8 \times 10^{17}$ $\mathrm{gs}^{-1}$. Magnetic moments weaker than those of AM Her stars is easier to drown by high accretion rates.

Even at lower accretion rates the magnetic nature of VY Scl could not be easy to detect. Circular polarization has been detected only in five IPs out of a total of more than 30, the ones that harbor white dwarfs with highest magnetic fields $\sim 2-8 M G$ (see Warner 1995). Since VY Scl stars could have fields lower than this value the detection of their circular polarization would be rather difficult. Indeed, Tapia (1981, quoted in Robinson et al. 1981) found the circular polarization of MV Lyr to be less than $0.13 \%$ and consistent with zero. This is comparable with the circular polarization of V795 He and LS Peg. In their low states, VY Scl are very weak X-ray sources which makes it difficult to find periodic signals. Probably the best time for detecting magnetic properties of these systems would be to observe them during the intermediates states.

If indeed VY Scl have magnetic moments $\mu \sim 10^{33} \mathrm{G} \mathrm{cm}^{3}$ they would be just highly accreting IPs and could not be AM Her star progenitors (see e.g. King \& Lasota 1991). This would mean that the distribution of magnetic moments is bi-modal.

In any case the fact that all VY Scl have orbital periods contained between 3 and 4 hours (see Fig. 2) remains a mystery. The same is true of the cause of mass-transfer fluctuations.

\section{References}

Buat-Ménard, V., Hameury, J.-M., \& Lasota, J.-P. 2001 A\&A, 369, 925

Hameury, J.M., Lasota, J.P., \& Dubus, G. 1999, MNRAS, 303, 39

Hameury, J.-M., Menou, K., Dubus, G., Lasota, J.-P., \& Huré, J.-M. 1998, MNRAS, 298, 1048

Hellier, C., Naylor, T. 1998, MNRAS, 295, L50 
Horne, K. 1999, in Magnetic Cataclysmic Variables, eds. K. Mukai, C. Hellier, ASP Conference Series, Vol. 157, p. 349

King, A.R., \& Lasota, J.-P. 1991, ApJ, 378, 674

Lasota, J.-P. 2001, NewAR, 45, 449

Lasota, J.-P., Hameury, J.-M. 2003, in preparation

Lasota, J.-P., Hameury, J.-M., Huré, J.-M. 1995, ApJ, 302, L29

Leach, R., Hessman, F.V., King, A.R., Stehle, R., \& Mattei, J. 1999, MNRAS, 305,225

Robinson, E.L., Barker, E.S., Cochran, A.L., Cochran, W.D., Nather, R.E. 1981, ApJ, 251, 611

Rodríguez-Gil, P., Casares, J., Martínez-Pais, I.G., Ignacio G., Hakala, P., \& Steeghs, D. 2001, ApJ, 548, L49

Rodríguez-Gil, P., Casares, J., Martínez-Pais, \& I.G. Hakala, P. 2002, in The Physics of Cataclysmic Variables and Related Objects, eds. B.T. Gänsicke, K. Beuermann, K. Reinch, ASP Conference Series, Vol. 261, P. 533

Patterson, J., Fenton, W.H., Thorstensen, J.R. (+ 15 co-authors) 2002, PASP, 114,1364

Sion, E.M. 1999, PASP, 111, 532

Sion, E. M. \& Urban, J. 2002, ApJ, 572, 456

Warner, B. 1995, Cataclysmic Variable Stars. Cambridge University Press, Cambridge 